\title{
Scaling of Stochasticity in Dengue Hemorrhagic Fever Epidemics
}

\author{
M. Aguiar ${ }^{1,2}$, B.W. Kooi ${ }^{3}$, J. Martins ${ }^{4}$, N. Stollenwerk ${ }^{1 *}$ \\ ${ }^{1}$ Centro de Matemática e Aplicações Fundamentais da Universidade de Lisboa \\ Avenida Professor Gama Pinto 2, 1649-003 Lisboa, Portugal \\ ${ }^{2}$ Fundação Ezequiel Dias, Serviço de Virologia e Riquetisioses \\ Laboratório de dengue e febre amarela \\ Rua Conde Pereira Carneiro 80, 30510-010 Belo Horizonte-MG, Brazil \\ ${ }^{3}$ Faculty of Earth and Life Sciences, Department of Theoretical Biology, Vrije Universiteit \\ De Boelelaan 1087, NL 1081 HV Amsterdam, The Netherlands \\ ${ }^{4}$ Department of Mathematics, School of Technology and Management, Polytechnic Institute of Leiria \\ Campus 2, Morro do Lena, Alto do Vieiro, 2411-901 Leiria, Portugal
}

\begin{abstract}
In this paper we analyze the stochastic version of a minimalistic multi-strain model, which captures essential differences between primary and secondary infections in dengue fever epidemiology, and investigate the interplay between stochasticity, seasonality and import. The introduction of stochasticity is needed to explain the fluctuations observed in some of the available data sets, revealing a scenario where noise and complex deterministic skeleton strongly interact. For large enough population size, the stochastic system can be well described by the deterministic skeleton gaining insight on the relevant parameter values purely on topological information of the dynamics, rather than classical parameter estimation of which application is in general restricted to fairly simple dynamical scenarios.
\end{abstract}

Keywords and phrases: dengue fever epidemiology, multi-strain model, external infections, deterministic skeleton, stochastic system

Mathematics Subject Classification: 92D30, 92B05, 93A30, 82C31

\section{Introduction}

Recently, we have investigated an epidemic multi-strain model motivated by dengue fever epidemiology, which shows deterministic chaos in wide parameter regions $[9,10]$. The addition of seasonal forcing, mimicking the vectorial dynamics, and a low import of infected individuals, which is realistic in the dynamics of infectious diseases epidemics showed complex dynamics and qualitatively a good agreement between empirical DHF monitoring data and the obtained model simulation [11].

Classical examples of chaos in epidemiological models are childhood diseases with extremely high infection rates so that a moderate seasonal forcing can generate Feigenbaum sequences of period doubling

\footnotetext{
*Corresponding author. E-mail: maira@ptmat.fc.ul
} 
bifurcations into chaos. In other infectious diseases much lower forces of infection have to be considered leading to further conceptual problems with noise affecting the system more than the deterministic part, leading even to critical fluctuations with power law behavior, when considering evolutionary processes of harmless strains of pathogens versus occasional accidents of pathogenic mutants [16]. Only explicitly stochastic models, of which the classical ODE models are mean field approximation versions, can capture the fluctuations observed in time series data [17].

In this paper, we investigate the role of dynamic noise in understanding epidemiological systems, such as dengue fever, by deriving a stochastic version of ordinary differential equations from Markov processes for discrete populations. Our model has the minimal degree of complexity to generate both primary and secondary dengue infections. The introduction of stochasticity is needed to explain the fluctuations observed in some of the available data sets, revealing a scenario where noise and complex deterministic skeleton strongly interact. For large enough population size, the stochastic system gives rise to the observed time series incidences. The classical parameter estimation and its application are generally restricted to fairly simple dynamical scenarios (where the statistical inference of parameter values are done via simple models such as the SIR dynamical model) and therefore a qualitative analysis of epidemiological data would have good chances to give insights into the relevant parameter values purely on topological information describing the type of long-term dynamics such as equilibrium, periodic solution, chaos and their dependency on parameters of the dynamics. These relevant parameter values can then be used for further refinement in formal parameter estimation.

\section{Modeling dengue fever epidemiology}

According to the estimates giving by [1], dengue infection is a leading cause of illness and death in the tropics and subtropics. More than one-third of the world's population are living in areas at risk of acquiring dengue infection and it is estimated that every year, there are $70-500$ million dengue infections, generating 36 million cases of dengue fever (DF) and 2.1 million cases of dengue hemorrhagic fever (DHF), with which without proper medical care the fatality rates can exceed $20 \%[20,30]$. There are four antigenically distinct but closely related dengue viruses, belonging to the family Flaviviridae, designated by DV-1, DV-2, DV-3, and DV-4. Infection by one serotype confers life-long immunity to only that serotype and a short period of temporary cross-immunity to a subsequent infection with other serotypes $[24,27,30]$. Field researchers in dengue have found that severe disease is $15-80$ times more likely in secondary then in primary infections and was positively associated with antibody dependent enhancement (ADE) of infection of mononuclear phagocytes. Infection by an antibody-virus complex suppresses innate immune responses, increasing intracellular infection and generating inflammatory citokines and chemokines that, collectively, result in enhanced disease $[8,12,24-26,28,30]$. Treatment of uncomplicated dengue cases is only supportive, and severe dengue cases require careful attention to fluid management and proactive treatment of hemorrhagic symptoms $[1,30]$. At present, there is no vaccine for dengue viruses, although several candidates are at various stages of development.

Dengue epidemiology dynamics is well known to be particularly complex with large fluctuations of disease incidences. Mathematical models describing the transmission of dengue viruses have focused on the ADE effect and temporary cross immunity trying to explain the irregular behavior of dengue epidemics. Besides the fact that disease propagation is an inherently stochastic phenomenon, dengue models are mainly expressed mathematically as a set of deterministic differential equations which are easier to analyze. The mean field approximation is a good approximation to be used in order to understand better the behavior of the stochastic systems in certain parameter regions, where the dynamics of the mean quantities are approximated by neglecting correlations. However, it is only stochastic, as opposed to deterministic, models that can capture the fluctuations observed in some of the available time series data. 


\section{The stochastic model}

The various multi-strain models currently investigated are essentially of SIR type. In the simple SIR epidemics without strain structure of the pathogens we have the following reaction scheme for the possible transitions from one to another disease related state, susceptibles $S$, infected $I$ and recovered $R$,

$$
\begin{gathered}
S+I \stackrel{\beta}{\longrightarrow} I+I \\
I \stackrel{\gamma}{\longrightarrow} R \\
R \stackrel{\alpha}{\longrightarrow} S
\end{gathered}
$$

for a host population of $N$ individuals, with contact and infection rate $\beta$, recovery rate $\gamma$ and temporary immunity rate $\alpha$. The deterministic ODE model

$$
\begin{aligned}
\dot{S} & =\alpha R-\frac{\beta}{N} \cdot I \cdot S \\
\dot{I} & =\frac{\beta}{N} \cdot I \cdot S-\gamma I \\
\dot{R} & =\gamma I-\alpha R
\end{aligned}
$$

describes in mean field approximation $\langle S \cdot I\rangle \approx\langle S\rangle \cdot\langle I\rangle$ the dynamics of the mean values, e.g. $\langle I\rangle:=$ $\sum_{S=0}^{N} \sum_{I=0}^{N} I p(S, I, t)$, where the initial values determine the time course of the system for all times. For more details on the calculations see e.g. [18].

For the SIR-model, the dynamics of probabilities in the form of a master equation [15] reads

$$
\begin{aligned}
\frac{d p(S, I, t)}{d t} & =\frac{\beta}{N}(S+1)(I-1) \quad p(S+1, I-1, t) \\
& +\gamma(I+1) \quad p(S, I+1, t) \\
& +\alpha(N-(S-1)-I) \quad p(S-1, I, t) \\
& -\left(\frac{\beta}{N} S I+\gamma I+\alpha(N-S-I)\right) \quad p(S, I, t)
\end{aligned}
$$

This process can be simulated by the Gillespie algorithm giving stochastic realizations. Only few stochastic processes can be solved explicitly, however, the mean field approximation is a good approximation to be used in order to understand the behavior of the stochastic systems in certain parameter regions.

Multi-strain dynamics are generally modeled more extended with SIR-type models, dividing the host population into susceptible, infected and recovered individuals with subscripts for the respective strains. The stochastic version of the multi-strain dengue model is now in complete analogy to the previously described SIR model, and the mean field ODE system for the multi-strain dengue model can be read from the following reaction scheme

$$
\begin{aligned}
S+I_{1} & \stackrel{\beta(t)}{\longrightarrow} I_{1}+I_{1} \\
S+I_{21} & \stackrel{\phi \beta(t)}{\longrightarrow} I_{1}+I_{21} \\
I_{1} & \stackrel{\gamma}{\longrightarrow} R_{1} \\
R_{1} & \stackrel{\alpha}{\longrightarrow} S_{1} \\
S_{1}+I_{2} & \stackrel{\beta(t)}{\longrightarrow} I_{12}+I_{2} \\
S_{1}+I_{12} & \stackrel{\phi \beta(t)}{\longrightarrow} I_{12}+I_{12} \\
I_{12} & \stackrel{\gamma}{\longrightarrow} R
\end{aligned}
$$


describing the transitions for first infection with strain 1 and secondary infection with strain 2, and for the reverse process, where the first infection is caused by strain 2 and the secondary infection is caused by strain 1 , the same reaction scheme can be used to describe the transitions by just changing labels. The demographic transitions are $S, I_{1}, I_{2}, R_{1}, R_{2}, S_{1}, S_{2}, I_{12}, I_{21}, R \stackrel{\mu}{\longrightarrow} S$ defining the system of two strains completely (for more information on the deterministic ODE system and its parametrization, see $[9,11]$ ). The parameter $\beta$ takes the seasonal forcing into account as a cosine function, $\beta(t)=\beta_{0}(1+\eta \cos (\omega t))$, where $\beta_{0}$ is the basic infection rate and $\eta$ is the degree of seasonality. The parameter $\gamma$ is the recovery rate, $\alpha$ is the temporary cross-immunity rate and $\phi$ is the ratio of secondary infection contribution to the force of infection. A low import factor is also included $(S \stackrel{\rho}{\longrightarrow} I)$ where $S$ can be any susceptible like $S, S_{1}$ or $S_{2}$ and $I$ respectively $I_{1}, I_{2}, I_{12}$ or $I_{21}$. In the simple SIR system, Eq. System (3.2), this gives $\dot{S}=\alpha R-\frac{\beta}{N} S(I+\rho N)$ etc.

We assume no epidemiological asymmetry between strains, i.e. infections with strain one followed by strain two or vice versa contribute in the same way to the force of infection. Here, the only relevant difference concerning disease transmissibility is that the force of infection varies accordingly to the number of previous infections the hosts have experienced. In a primary infection the individuals transmit the disease with a force of infection $\frac{\beta I}{N}$ whereas in a secondary infection the transmission is given with a force of infection $\frac{\phi \beta I}{N}$ where $\phi$ can be larger or smaller than unit, i.e. increasing or decreasing the transmission rate. For the stochastic simulations the parameter values are given in Table 1, if not otherwise explicitly stated.

TABLE 1. Parameter set, rates given in units per year, ratio without unit

\begin{tabular}{llll}
\hline Par. & Description & Values & Ref \\
\hline$\mu$ & new born susceptible rate & $1 / 65 y$ & {$[22]$} \\
$\gamma$ & recovery rate & $52 y^{-1}$ & {$[6,30]$} \\
$\beta_{0}$ & infection rate & $\in[\gamma, 2 \gamma]$ & {$[14]$} \\
$\alpha$ & temporary cross-immunity rate & $2 y^{-1}$ & {$[21,27]$} \\
$\phi$ & ratio of contrib. to force of inf. & 0.9 & {$[11]$} \\
$\eta$ & degree of seasonality & $\in[0,0.2]$ & {$[11]$} \\
$\ln (\rho)$ & import factor & $\in[-17,-15.5]$ & {$[11]$} \\
& & & \\
\hline
\end{tabular}

The first recorded epidemic of DHF in Thailand (population of approximately 66 million people [29]) was in 1958 [30]. The co-circulation of all four dengue serotypes and their capacity to produce severe dengue disease was demonstrated as early as 1960 in Bangkok, Thailand [23]. DHF occurred first only in Bangkok, but was disseminated to the whole region during the $1970 s[3,19,23]$. Physicians in Thailand are trained to recognize and treat dengue fever and practically all cases of DHF and DSS are hospitalized. A system for reporting communicable diseases including DHF/DSS was considered fully installed in 1974 and the data bank of DHF and DSS is available at the Ministry of Public Health, Bangkok [19]. For such a data scenario, models that are able to generate both primary and secondary infection cases (with a different strain), without the need of considering sensitivity of the dynamics of different co-circulating dengue serotypes, have shown a good qualitative agreement between empirical data and model output [11] just by combining ADE and temporary cross-immunity.

The inspection of the available DHF incidence data in Thailand shows a smooth behavior with a well defined maximum each year of irregular height for the Northern Provinces as opposed to the Central and Southern Provinces where the data is very noisy linked with a low endemicity of DHF cases. In [11] the Province of Chiang Mai was taken as a case study and the empirical DHF incidence data was compared with the time series simulation obtained from the seasonal multi-strain model with import giving qualitatively a very good result, suggesting that the used parameter set could be the starting set 
for a more detailed parameter estimation procedure. However, in order to describe the noisy dynamics in Bangkok for example the introduction of stochasticity is even more important.

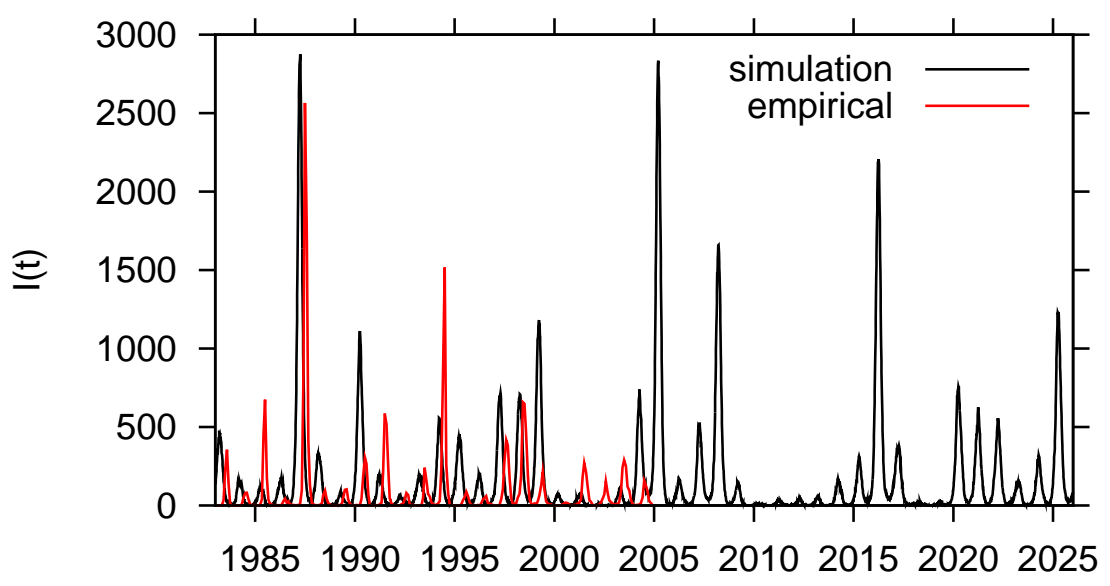

a)

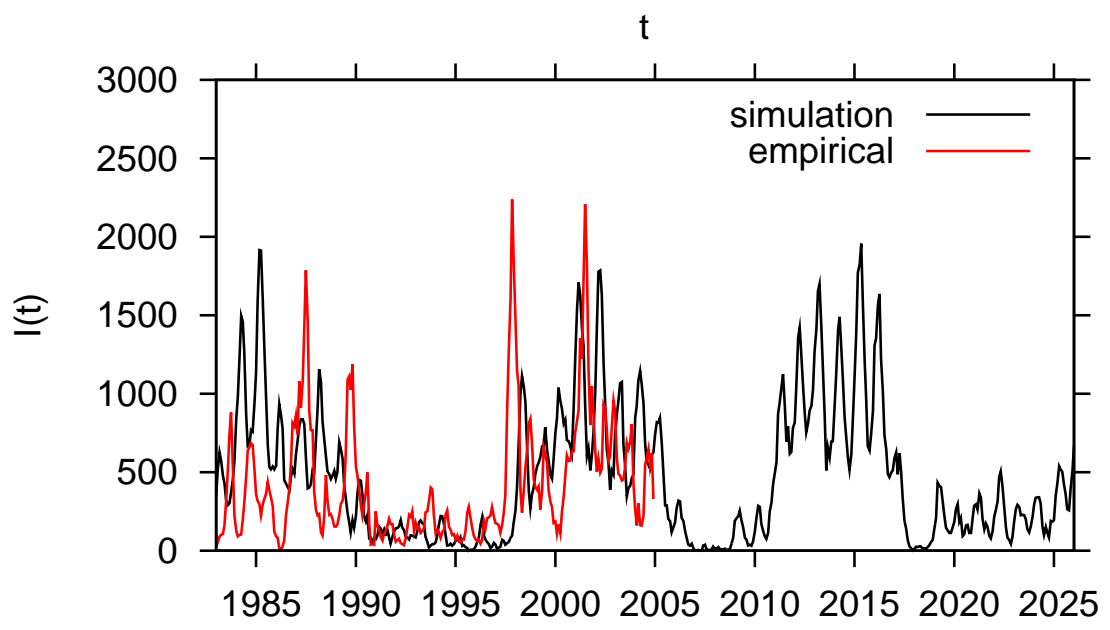

b)

$\mathrm{t}$

Figure 1. Empirical DHF incidence data (in red) matched with one stochastic realization (in black) for the seasonal multi-strain dengue model with import. In a) we show the incidences for Chaing Mai. For the stochastic simulation the infection rate is $\beta_{0}=2 \gamma$, the degree of seasonality $\eta=0.2$ and the import $\ln (\rho)=-15.7$. In b) we show the incidences for Bangkok. For the stochastic simulation the infection rate is $\beta_{0}=1.1 \cdot \gamma$, the degree of seasonality $\eta=0.06$ and the import $\ln (\rho)=-16.9$. The other parameter values are listed in Table 1.

In Fig. 1a) we show the DHF incidences for Chaing Mai (in red), one of the Northen Provinces of Thailand, with population size $N=1650000$ [22] compared with one stochastic realization for the multistrain dengue model (in black) where the infection rate $\beta_{0}=2 \gamma$, the degree of seasonality $\eta=0.2$ and the import $\ln (\rho)=-15.7$. In Fig. 1b) the DHF incidences for Bangkok (in red), the capital of Thailand, with population size $N=6600000$ [22] is compared with one stochastic realization for the multi-strain dengue model (in black) where the infection rate $\beta_{0}=1.1$, the degree of seasonality $\eta=0.06$ and the import 
$\ln (\rho)=-16.9$. The stochastic approach is able to describe both types of the dynamics, the smooth data with a well defined maximum each year of irregular height, found in the high endemic regions of Thailand, e.g. in the Chiang Mai Province (see Fig. 1a)) and also the noisy data found mainly in low endemic regions of Thailand, e.g. in Bangkok (see Fig. 1b)).

Using the same values for the biological parameters given in [11], the fluctuations observed in the empirical data were qualitatively well described in the stochastic model. Under population noise low seasonal forcing was needed to represent the DHF incidences in the Province of Chiang Mai as opposed to the deterministic approach where the combination seasonality and import showed complex dynamics. For the representation of the DHF incidences in Bangkok, only the stochastic model could capture the noisy behavior where even lower seasonal forcing was needed as well a low infection rate. A value of $\beta_{0}=1.1 \cdot \gamma$ is qualitatively in good agreement with the data (see Fig. 1b)) and hence the import factor $\rho$ is here, more than the direct infectivity $\beta$, the driving force of the epidemic pattern in such a low endemic region.

\section{The role of import}

In our model, the parameter $\rho$ is the import factor, related with the possibility of an individual to get infected outside the studied population and then bring the infection into the population that this individual belongs to, mimicking the imported cases of the disease in a defined population. Equivalently an infected visitor to the region under consideration who passes the infection to a susceptible in the population of size $N$ has the same effect on the studied population. Hence, we do not need to distinguish this two scenarios of susceptibles traveling outside or infected traveling inside the region under consideration. In total, this captures the imported infection that comes from an external source.

In Figure 2 we present the bifurcation diagram comparison between the non-seasonal model, the nonseasonal model with import and the seasonal model with import. The bifurcation diagram for the original non-seasonal model previously studied in $[9,10]$, shows two chaotic windows, a new chaotic window in a $\phi$ region where the ratio of secondary infection contribution to the force of infection is smaller than 1 and also the classical chaotic window found previously in $[9,14]$, where the ratio of secondary infection contribution to the force of infection is much larger than 1, actually around $\phi=3$ (see Fig. 2a)). The bifurcation diagram for the non-seasonal model described in [9] with addition of a low import of infected shows that the addition of import in such a system removes the complex dynamics in the region of $\phi$ larger 1 where the stable limit cycle is the unique attractor (see Fig. 2b)). The bifurcation diagram for the seasonal model with import described in [11], shows that the combination of seasonality and import brings back the chaotic attractors for even larger parameter regions (see Fig. 2b)).

These results are important since we expect complex dynamics to explain the fluctuations observed in empirical data, when the ratio of secondary infection contribution to the force of infection could be slightly smaller or larger 1 , not needing to restrict the ADE effect to one or another region in parameter space.

In the multi-strain dengue model the susceptible individuals without a previous dengue infection can get infected with two different infection rates, due to the ADE effect leading to severe disease requiring hospitalization. Individuals in the first infection would then transmit more than individuals in the secondary infection. For more information on the parametrization of the two-strain dengue model, see $[9,11]$. Individuals infected for the first time become recovered and life long immune to that strain and, after a period of temporary cross immunity, are again susceptible, however with an experienced previous infection. The second infection can only happen with a different strain. Individuals infected for the second time would more likely need to be hospitalized due to the severity of the disease. They recover and then become life long immune to the other strain. There is no epidemiological asymmetry between strains, i.e. infections with strain one followed by strain two or vice versa contribute in the same way to the force of infection, so the notion of two different strains is enough to describe differences between primary infections, often asymptomatic, and secondary infection, associated with the severe form of the disease. The death rates coming out of all classes go into the class of susceptible without experiencing 


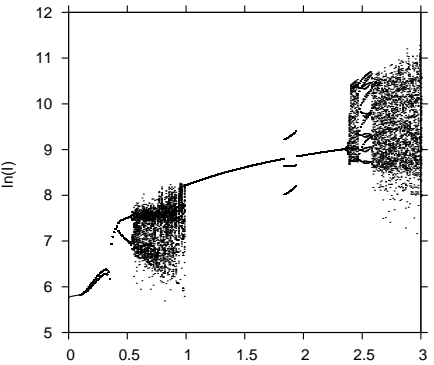

a) $\phi$

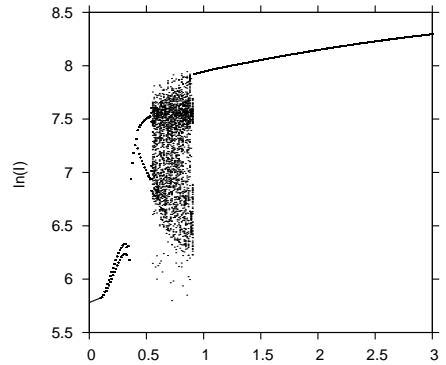

b)

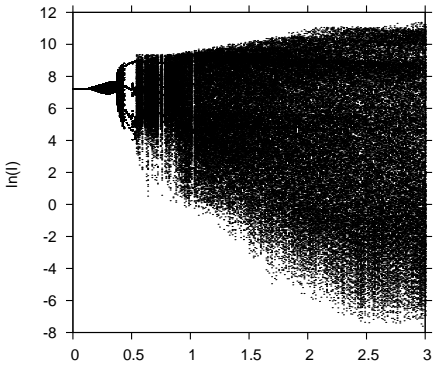

c)

FiguRE 2. Bifurcation diagram comparison between the non-seasonal model, the nonseasonal model with import and the seasonal model with import. Here, the local maxima of the logarithm of the total number of infected $\ln (I)=\ln \left(I_{1}+I_{2}+I_{12}+I_{21}\right)$ are plotted against the ratio of the contribution of the secondary infections $\phi$ to the force of infection. In a) we show the bifurcation diagram for the original non-seasonal model previously studied in $[9,10]$, in b) we show the bifurcation diagram for the non-seasonal model described in [9] with addition of a low import of infected and in c) we show the bifurcation diagram for the seasonal model with import described in [11].

previous dengue infection as a birth rate. Since the demographic events often occur at a much slower rate than the infection, the disease has to be necessarily maintained by constant external infections to avoid the repeated stochastic disease extinction and re-introduction [2,13].

For the deterministic system a torus bifurcation $T R$ was the first bifurcation happening for a region of import factor $\ln (\rho)=-15.85$ (see Fig. 3a)). Lyapunov exponents were calculated along the trajectory and the Lyapunov spectrum is shown in Fig. 3b), where two dominant zero Lyapunov exponents at $\ln (\rho)=-15.85$ shows a quasi-periodicity (for instance on a torus). The appearance of this bifurcation for $\ln (\rho)=-15.84$ is also predicted by AUTO [7]. In order to illustrate the infected dynamics on the deterministic approach we show the time series and its state space plot in Fig. 3c) and Fig. 3d).

Using the same parameter values as used for the deterministic simulations, the quasi-periodicity becomes more irregular resembling a chaotic behavior in the stochastic modeling approach. Figure 4a) shows one stochastic realization for the multi-strain dengue model which could describe very well the dynamics of the DHF incidences in Chiang Mai Province, North of Thailand (see Fig. ??a)). Such pattern would be most likely be described as being the chaotic transients towards the quasi-periodic torus of the deterministic skeleton (see 4b)) that got stabilized due to the noise.

\section{Scaling of stochasticity}

It is known that stochastic simulations, using a finite size population, involve extinction phenomena operating through demographic stochasticity which acts drastically on small populations, as opposed for the deterministic models that do not handle extinction through population noise, leading to populations with very few individuals or even fractions of individuals. In Fig. 5 we compare the deterministic and stochastic dynamics and we see that the magnitude of stochastic fluctuations decreases when the population size increases. We compare the interplay between stochastic model and deterministic skeleton in 4 different systems sizes. In Fig. 5a) we show the stochastic and deterministic system's interaction for a system where the population size $N=1.65 \cdot 10^{6}$, mimicking the Province of Chiang Mai. The system with small population size shows very large fluctuations around the deterministic skeleton, hence an extreme noise amplification. In Fig. 5b) we show the stochastic and deterministic system's interaction for a system where the population size $N=6 \cdot 10^{6}$, as it would be for the North of Thailand. In Fig. 5c) the system has the population size of Thailand, $N=66 \cdot 10^{6}$ and in Fig. 5 d) a larger system size, where the population of some countries surrounding Thailand, for instance Burma, Laos, Vietnam and 

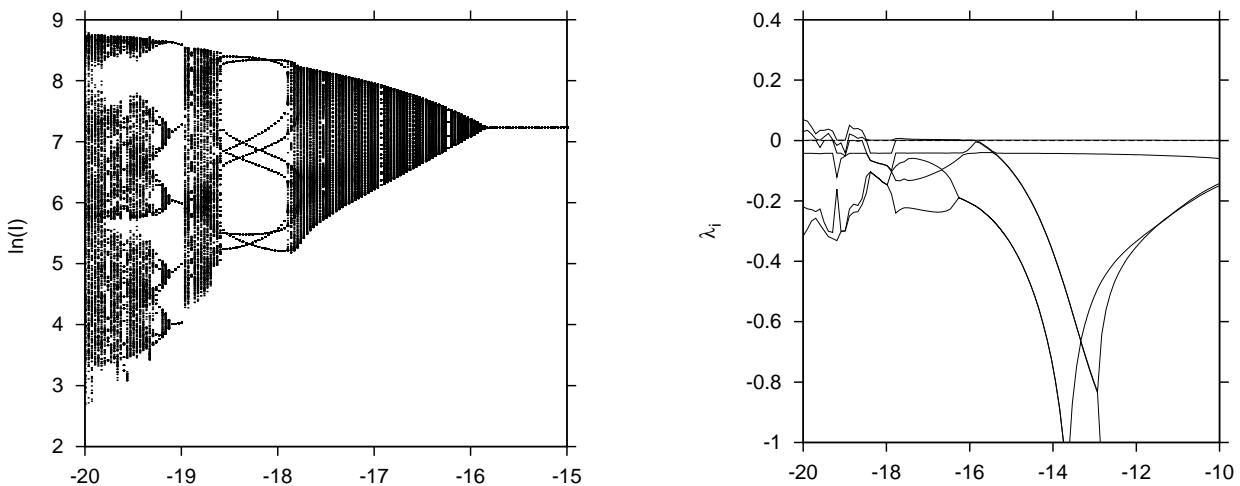

a)

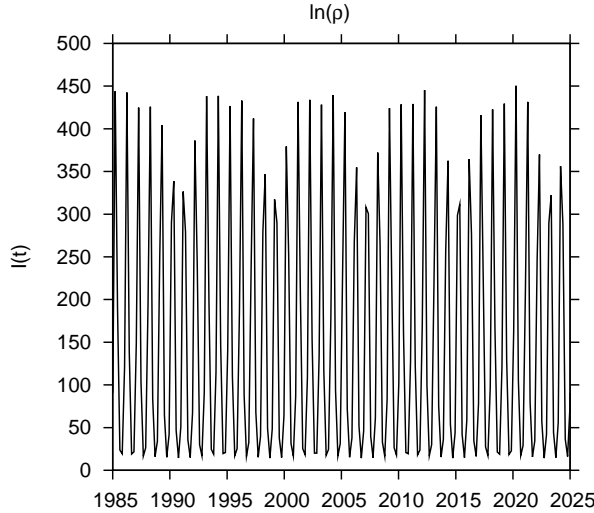

c) b)

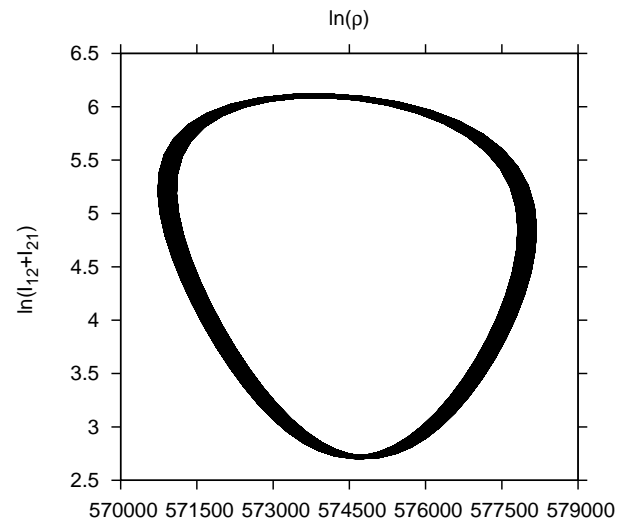

d)

Figure 3. Numerical simulations for the province of Chiang Mai with population size $N=1650000$. The parameter values are fixed as follows: Temporary cross immunity $\alpha=2 y^{-1}$, infection rate $\beta_{0}=2 \gamma$, recovery rate $\gamma=52 y^{-1}$, ratio of the contribution of the secondary infections to the force of infection $\phi=0.9$, seasonal forcing $\eta=0.2$ and import $\ln (\rho)=-15.85$. In a) the bifurcation diagram varying the import parameter is shown. In b) the Lyapunov spectrum varying the import parameter, in c) the time series simulation and in d) the state space plot are shown. The torus attractor is visible here.

Cambodia, were counted together giving a system where the population size is $N=230 \cdot 10^{6}$. For such large system, the stochastic fluctuations follow quite well the deterministic approach, where the noise is not much amplified anymore.

We see that the magnitude of the stochastic fluctuations decreases when the population size increases and more importantly, that for large enough population size, the stochastic system can be well described by the deterministic skeleton, where the essential dynamics are captured.

\section{Conclusions}

Multi-strain dynamics are generally modeled with SIR-type models, dividing the host population into susceptible, infected and recovered individuals with subscripts for the respective strains. We have considered the stochastic version of a multi-strain model with a minimal degree of complexity to generate both primary and secondary infections, motivated by dengue fever epidemiology. Besides the fact that disease propagation is an inherently stochastic phenomenon, dengue models are mainly expressed mathematically as a set of deterministic differential equations which are easier to analyze, however, it is only stochastic, 


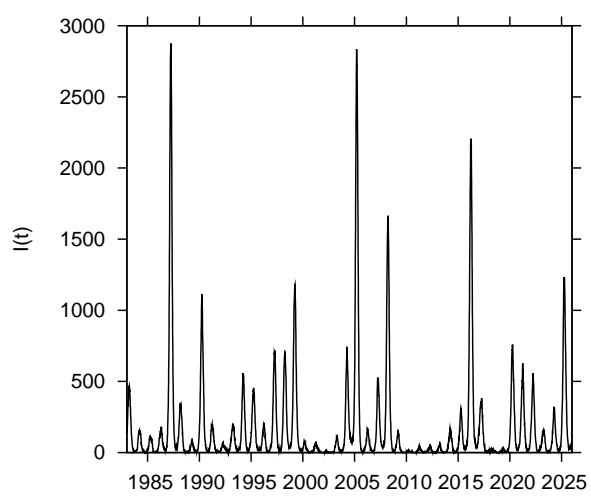

a)

FIGURE 4. Stochastic and deterministic system's comparison. In a) One stochastic realization for a population size $N=1650000$, temporary cross immunity $\alpha=2 y^{-1}$, infection rate $\beta_{0}=2 \gamma$, recovery rate $\gamma=52 y^{-1}$, ratio of the contribution of the secondary infections to the force of infection $\phi=0.9$, seasonal forcing $\eta=0.2$ and import $\ln (\rho)=$ -15.85. In b) the deterministic time series simulation as shown in Fig. $3 \mathrm{c}$ ), scaled up to be properly compared with the stochastic time series simulation in Fig. 4a).

as opposed to deterministic, models that can capture the fluctuations observed in some of the available time series data.

For the minimalistic multi-strain dengue model, the individuals can be susceptibles without a previous dengue infection, infected and recovered for the first time, susceptible with an experienced previous infection and infected for the second time, now with a different strain, and more likely been hospitalized due to the ADE effect leading to severe disease. Since the demographic events often occur at a much slower rate than the infection, the disease has to be necessarily maintained by the import of external infections to avoid the repeated stochastic disease extinction and re-introduction.

The stochastic realizations of infected in time were obtained by the Gillespie algorithm $[4,5]$. By considering stochasticity and external infections, we have shown that the introduction of stochasticity was needed to explain the fluctuations observed in some of the available data sets, revealing a scenario where noise and complex deterministic skeleton strongly interact. For large enough population size, the stochastic system could be well described by the determisnistic skeleton, where the essential dynamics are captured, gaining insight into the relevant parameter values purely on topological information of the dynamics.

Understanding the dynamics of stochastic populations, and how they interact with the deterministic components of epidemiological models have maximum benefit on the practical predictability of the dynamical system by analyzing the available epidemiological data via mathematical models, since the classical parameter estimation and its application are generally restricted to fairly simple dynamical scenarios. The ability to predict the future dengue outbreaks via mathematical models would provide a tool to guide policies of prevention and control of the dengue virus transmission, including the implementation of vaccination programs when the dengue fever vaccine will be accessible. 

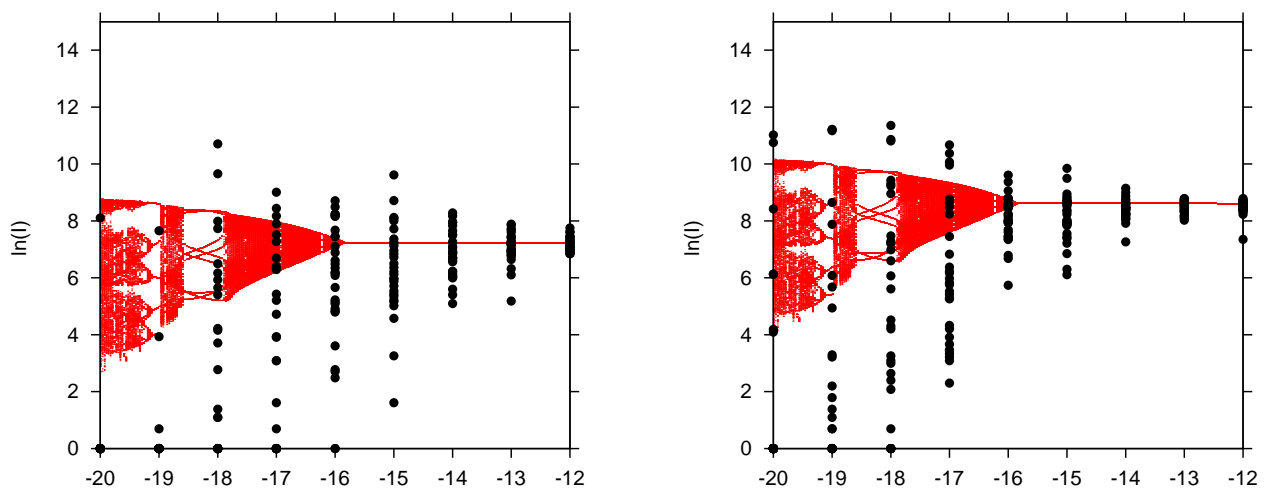

a)

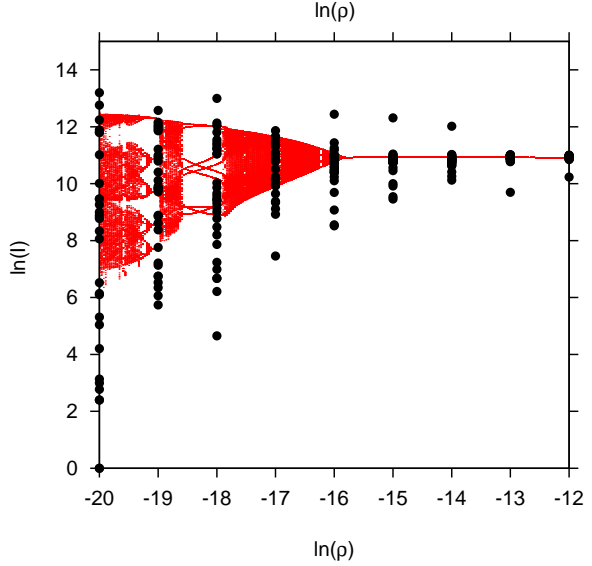

b)

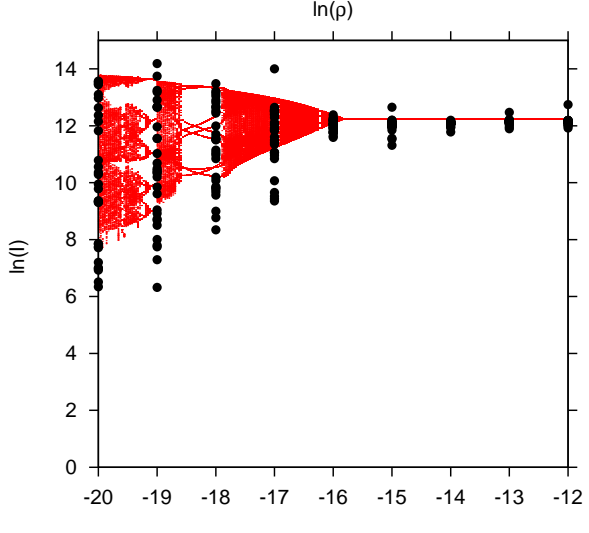

d)

$\ln (\rho)$

FIGURE 5. Stochastic and deterministic system's interaction. For the same parameter values used in Fig. 4, we show the bifurcation diagram for the import parameter for different population sizes $N$. In red the deterministic model and in black the stochastic model. In a) the Chiang Mai population size $N=1.65 \cdot 10^{6}$, in b) North of Thailand population size $N=6 \cdot 10^{6}$, in c) Thailand population size $N=66 \cdot 10^{6}$ and in d) a larger system size, where the population of some countries surrounding Thailand, for instance Burma, Laos, Vietnam and Cambodia, were counted together giving a system where the population size is $N=230 \cdot 10^{6}$.

Acknowledgements. We would like to thank Bernard Cazelles, Ecole Normale Supérieure, Paris, France for providing the available DHF incidence data in Thailand. Yoshiro Nagao, Osaka University Graduate School of Medicine in Japan, and Katia Koelle, Biology Department of Duke University in the USA for providing the extended Thailand DHF incidence data, Francisco Lemos and Sonia Diniz, Belo Horizonte, Minas Gerais, and Scott Halstead, Bethesda, Maryland, for detailed information about dengue epidemiology. Maíra Aguiar also acknowledges the financial support from the FCT grant with reference SFRH/BD/43236/2008. This work has been further supported by the European Union under FP7 in the EPIWORK project and the Portuguese FCT project PTDC/MAT/115168/2009.

\section{References}

[1] Centers for Disease Control and Prevention. Dengue, (2011). Retrieved from http://www.cdc.gov/dengue/

[2] D. Alonso, A. McKane, M. Pascual. Stochastic Amplification in Epidemics. Journal of the Royal Society Interface, (2006), 4, 575-582.

[3] D. J. Gubler. Epidemic dengue/dengue hemorrhagic fever as a public health, social and economic problem in the 21st century. Trends in Microbiology, (2002), 10, 100-103. 
[4] D. T. Gillespie. A general method for numerically simulating the stochastic time evolution of coupled chemical reactions. Journal of Computational Physics, (1976), 22, 403-434.

[5] D. T. Gillespie. Monte Carlo simulation of random walks with residence time dependent transition probability rates. Journal of Computational Physics, (1978), 28, 395-407.

[6] J. D. Gubler, W. Suharyono, R. Tan, M. Abidin, A. Sie. Viraemia in patients with naturally acquired dengue infection. Bull. World Health Organ., (1981), 59, 623-630.

[7] J. E. Doedel, B. Oldeman. AUTO 07P - Continuation and bifurcation software for ordinary differential equations. Technical Report: Concordia University, Montreal, Canada, (2009). Retrieved from http://indy.cs.concordia.ca/auto/

[8] J. S. Mackenzie, D. J. Gubler, L. R. Petersen. Emerging flaviviruses: the spread and resurgence of Japanese encephalitis, West Nile and dengue viruses. Nature Medicine Review, (2004), 12, S98-S109.

[9] M. Aguiar, B. W. Kooi, N. Stollenwerk. Epidemiology of Dengue Fever: A Model with Temporary Cross-Immunity and Possible Secondary Infection Shows Bifurcations and Chaotic Behaviour in Wide Parameter Regions. Math. Model. Nat. Phenom., (2008), 4, 48-70.

[10] M. Aguiar, N. Stollenwerk, B. W. Kooi. Torus bifurcations, isolas and chaotic attractors in a simple dengue model with ADE and temporary cross immunity. International Journal of Computer Mathematics, (2009), 86, $1867-1877$.

[11] M. Aguiar, S. Ballesteros, B. W. Kooi, N. Stollenwerk. The role of seasonality and import in a minimalistic multi-strain dengue model capturing differences between primary and secondary infections: complex dynamics and its implications for data analysis. Accepted for publication in Journal of Theoretical Biology, (2011).

[12] M. G. Guzmán et al. Dengue: a continuing global threat. Nature Reviews Microbiology, (2010), 8, S7-S16.

[13] M. J. Keeling, J. V. Ross. On methods for studying stochastic disease dynamics. Journal of the Royal Society Interface, (2008), 5, 171-181.

[14] N. Ferguson, R. Anderson, S. Gupta. The effect of antibody-dependent enhancement on the transmission dynamics and persistence of multiple-strain pathogens. Proc. Natl. Acad. Sci. USA, (1999), 96, 790-94.

[15] N. G. van Kampen. Stochastic Processes in Physics and Chemistry. (North-Holland, Amsterdam, 1992).

[16] N. Stollenwerk, V. A. A. Jansen. (2003, b). Evolution towards criticality in an epidemiological model for meningococcal disease. Physics Letters A, (2003, b), 317, 87-96.

[17] N. Stollenwerk, M. C. J. Maiden, V. A. A. Jansen, V.A.A. Diversity in pathogenicity can cause outbreaks of menigococcal disease. Proc. Natl. Acad. Sci. USA, (2004), 101, 10229-10234.

[18] N. Stollenwerk, V. V. A. Jansen. Population biology and criticality (Imperial College Press, London, 2010).

[19] O. Chareonsook et al. Changing epidemiology of dengue hemorrhagic fever in Thailand. Epidemiol. Infect., (1999), $122,161-166$.

[20] Pediatric Dengue Vaccine Initiative. International Vaccine Institute (IVI). Global Burden of Dengue, (2011). Retrieved from http://www.pdvi.org/about_dengue/GBD.asp

[21] Pers comm.: Francisco Lemos, Secretaria de Estado de Saúde de Minas Gerais, Brazil; Sônia Diniz, Fundação Ezequiel Dias, Minas Gerais, Brazil and Scott Halstead, Pedriatic Dengue Vaccine Initiative, Maryland, USA.

[22] United Nations Population Division World Urbanization Prospects: The 2009 Revision Population Database, (2011). Retrieved from http://www.un.org/esa/population/unpop.htm

[23] S. B. Halstead et al. Dengue and chikungunya virus infection in man in Thailand, 1962-1964. V. Epidemiologic observations outside Bangkok. Am. J. Trop. Med. Hyg., (1969), 18, 1022-33.

[24] S. B. Halstead. Antibody-dependent Enhancement of Infection: A Mechanism for Indirect Virus Entry into Cells. Cellular Receptors for Animal Viruses, 28, Chapter 25, 493-516. (Cold Spring Harbor Laboratory Press, 1994).

[25] S. B. Halstead. Immune enhancement of viral infection. Progress in Allergy, (1982), 31 301-364.

[26] S. B. Halstead. Neutralization and antibody-dependent enhancement of dengue viruses. Advances in Virus Research, (2003), 60, 421-467.

[27] S. Matheus et al. Discrimination between Primary and Secondary Dengue Virus Infection by an Immunoglobulin G Aviditnoy Test Using a Single Acute-Phase Serum Sample. Journal of Clinical Microbiology, (2005), 43, 2793-2797.

[28] W. Dejnirattisai et al. Cross-Reacting Antibodies Enhance Dengue Virus Infection in Humans. Science, (2010), 328, $745-748$.

[29] Wikipedia contributors. Wikipedia, The Free Encyclopedia. Provinces of Thailand, (2011). Retrieved from http://en.wikipedia.org/wiki/Provinces_of_Thailand

[30] World Health Organization. Dengue and Dengue Hemorrhagic Fever, Fact sheet 117, (2009). Retrieved from http://www.who.int/mediacentre/factsheets/fs117/en/

[31] Y. Nagao, K Koelle. Decreases in dengue transmission may act to increase the incidence of dengue hemorrhagic fever. Proc. Natl. Acad. Sci, (2008), 105, 2238-2243. 